\title{
THE FORCING METHOD AND THE UPPER SEMILATTICE OF HYPERDEGREES
}

\author{
BY \\ S. K. THOMASON( ${ }^{(1)}$
}

1.1. Introduction. The method of forcing was invented by Cohen [C] for application to set theory. Feferman $[F, \S 2]$ has adapted the Cohen techniques to first-order number theory. With Feferman's adaptation it is possible directly to obtain theorems concerning the existence of sets of natural numbers with prescribed arithmetical properties. (An arithmetical property is one expressible by a formula $F\left(X_{1}, \ldots, X_{n}, x_{1}, \ldots, x_{m}\right)$ of second-order number theory having no bound set variables.) Feferman's basic methods, applied with considerable ingenuity, suffice to prove some theorems referring to nonarithmetical properties of sets; this has been done by Feferman [F, §2], Addison [ $\left.\mathrm{A}_{2}\right]$, Hinman $[\mathrm{H}]$, and others. It seems, however, that the basic method has limited nonarithmetical applicability. This fact is not surprising; one should not expect the power of the forcing method, as applied to a particular language, to extend much beyond the properties expressible in that language. Thus one is led to attempt to define forcing for stronger languages. Of course, set theory is itself such a stronger language. But in order to get good upper bounds on the "computational complexity" of sets constructed by forcing techniques, it is necessary to use languages which are as weak as possible. Moreover, the use of the language of set theory introduces unpleasant complications regarding consistency, absoluteness, etc. Presumably motivated by these considerations, Feferman in [F, §3] extended forcing to a language of ramified analysis.

This paper comprises two parts. In the first we shall extend Feferman's notion of number-theoretic forcing to a stronger language. This construction is rather complicated, and occupies $\$ \S 1.2-1.5$. In $\$ 1.6$, the method will be used to deduce the existence of a pair of sets of numbers possessing a rather strong "incomparability property," namely they are both hyperarithmetic in Kleene's set $\boldsymbol{O}$ of constructive ordinal notations, yet neither is arithmetically definable from the hyperjump of the other. Our original program was to investigate the structure of the upper semilattice of hyperdegrees, and the outcome of this investigation, using

Presented to the Society, in part, June 19, 1965 under the title Hyperarithmetic incomparability; received by the editors May 20, 1966 and, in revised form, October 3, 1966.

( $\left.{ }^{1}\right)$ This paper is derived from the author's doctoral thesis (Cornell University, 1966). The author wishes to express his sincere gratitude to Professors G. E. Sacks and Anil Nerode for their guidance and suggestions. Some suggestions of the referee have been adopted, resulting, it is hoped, in a clearer presentation. The research was supported in part by the National Science Foundation of the U. S. A. 
the forcing method of the first part and minor variations thereof, is reported in the second part. In particular, we consider the following problems: 1. Given a nonhyperarithmetic set $B$, construct a set $A$ whose hyperdegree is incomparable with that of $B$. 2. Given a partially ordered set $P$, embed $P$ in the hyperdegrees, preserving the ordering of $P$ and as much as possible of the join and meet structure. 3. Given a set $B$ of hyperdegree greater than that of Kleene's $\boldsymbol{O}$, construct a set $\boldsymbol{A}$ such that $O^{A} \equiv_{h} B$. 4. Construct hyperarithmetically incomparable sets whose hyperdegrees have as least upper bound the hyperdegree of $\boldsymbol{O}$.

Most of the results were announced in $\left[\mathrm{T}_{1}\right],\left[\mathrm{T}_{2}\right],\left[\mathrm{T}_{4}\right]$.

The reader will be assumed to have some knowledge of the theory of hyperarithmetic sets, as developed by Kleene $\left[\mathrm{K}_{1}\right],\left[\mathrm{K}_{2}\right]$ and Spector [S], and of the forcing method as presented by Feferman $[\mathrm{F}, \S \S 1,2] . N=\{0,1, \ldots\}$ is the set of "numbers." Unless otherwise specified, "set" means "set of numbers." The ordered pair $\langle a, b\rangle$ is just the number $2^{a+1} \cdot 3^{b+1}$, similarly for triples, etc. $A+B$ is the set-theoretic union, and $A \cup B$ is always the recursively disjoint union; thus $A \cup B=\left\{2^{a+1} \mid A \in A\right\}+\left\{3^{b+1} \mid b \in B\right\}$; and similarly for infinite sums and unions. Whenever it seems convenient, sets are identified with their representing functions, and predicates and functions with their graphs. The $e$ th function partial recursive in $A$ is denoted by $e^{A}$.

1.2. Arithmetical closure conditions. A set $B$ is said to be defined by closure conditions arithmetical in the set $A$ if $x \in B \leftrightarrow(X)[S(X) \rightarrow x \in X]$, where $S(X)$ is arithmetical in $A$ and is a conjunction of closure conditions. It is evident in this case that $B$ is $\Pi_{1}^{1}$ in $A$; this remains true even if $S(X)$ is only $\Sigma_{1}^{1}$ in $A$. For example, the hyperjump $A^{\prime}$ of $A$ is defined to be the smallest set satisfying:

1. $\langle 1,2\rangle \in A^{\prime}$.

2. $\langle 1, a\rangle \in A^{\prime} \rightarrow\left\langle a, 2^{a}\right\rangle \in A^{\prime}$.

3. $(n)\left(\left\langle e^{A}(n), e^{A}(n+1)\right\rangle \in A^{\prime}\right) \rightarrow(n)\left(\left\langle e^{A}(n), 3 \cdot 5^{e}\right\rangle \in A^{\prime}\right)$.

4. $\langle a, b\rangle \in A^{\prime} \quad \&\langle b, c\rangle \in A^{\prime} \rightarrow\langle a, c\rangle \in A^{\prime}$.

The above constitute a definition of $A^{\prime}$ by closure conditions (uniformly) arithmetical in $A . A^{\prime}(x, y)$ is usually written $x<o^{A} y$, but we shall use the simpler notation as much as possible; $N^{\prime}$ will do the ordering job usually performed by $<\boldsymbol{o}$. (This definition of $A^{\prime}$ is essentially that of Kreider and Rogers [KR, p. 332].)

If $B$ is defined by closure conditions arithmetical in $A$, then one may think of the conditions as generating the elements of $B$ one at a time, in a well-ordered manner, probably extending into the transfinite. A general lemma would say that the idea of order of generation can always be made explicit in a manner faithful to the intuitive notion, and in such a way that $(\lambda x y)$ ( $x$ is generated before $y)$ is arithmetical in $A^{\prime}$. Since a precise statement of such a lemma would be rather opaque, we shall be content with two special cases: the following Lemma 1, and Lemma 3 of $\$ 1.3$. We do not give the proof of Lemma 1, since it is similar to that of Lemma 3, but simpler. 
Lemma 1. There is a partial function $\Phi$, whose graph is $\Pi_{1}^{1}$ in $A$, which maps a subset of $Q^{A}$ (a set of $A$-notations $\Pi_{1}^{1}$ in $A$, well ordered by $A^{\prime}$, and containing $a$ unique notation for each ordinal less than $\omega_{1}^{A} ; c f$. [FS]) onto $A^{\prime}$ such that if

$$
\Phi(a)=\langle x, y\rangle
$$

then one of the following holds:

1. $x=1 \& y=2$.

2. $y=2^{x} \&(E b)\left(A^{\prime}(b, a) \& \Phi(b)=\langle 1, x\rangle\right)$.

3. $(E e)(E n)\left[y=3 \cdot 5^{e} \& x=e^{A}(n) \&(z)(E b)\left(A^{\prime}(b, a) \& \Phi(b)=\left\langle e^{A}(z), e^{A}(z+1)\right\rangle\right)\right]$.

4. $(E z)(E b)(E c)\left[A^{\prime}(b, a) \& A^{\prime}(c, a) \& \Phi(b)=\langle x, z\rangle \& \Phi(c)=\langle z, y\rangle\right]$.

The correspondence between the alternatives of Lemma 1 and the closure conditions defining $A^{\prime}$ should be evident. The reader should satisfy himself that Lemma 1 is indeed a special case of the proposed general lemma.

1.3. Forcing. $\mathscr{L}$ is the language of elementary number theory, with disjunction, negation, and existential quantification as logical constants. As nonlogical constants, $\mathscr{L}$ has numerals ${ }^{\top} \mathbf{0}^{\top},{ }^{\top} \mathbf{1}^{\prime}, \ldots$, and predicate constants representing addition, multiplication, equality, and the successor operation. Forcing will be defined for the language $\mathscr{L}^{*}$ obtained from $\mathscr{L}$ by adding pairs $\boldsymbol{A}_{k}$ and $\boldsymbol{A}_{k}^{\prime}(k \in N)$ of binary predicate letters as nonlogical constants. (In [F, §2] Feferman defined forcing for essentially the language obtained from $\mathscr{L}$ by adding only the letters $\boldsymbol{A}_{\boldsymbol{k}}$.) $\boldsymbol{A}$ and $\boldsymbol{B}$ will stand for any of the letters $\boldsymbol{A}_{\boldsymbol{k}}$; if $\boldsymbol{A}$ is standing for $\boldsymbol{A}_{\boldsymbol{k}}$ then $\boldsymbol{A}^{\prime}$ will stand for $\boldsymbol{A}_{k}^{\prime}$. $\boldsymbol{A}$ is to be thought of as (the representing predicate of the representing function of) an unspecified set $A$, and $A^{\prime}$ as the hyperjump of $A$. A set of conditions is a finite set of sentences of $\mathscr{L}^{*}$ of the form $A\left(\Gamma^{\top} \boldsymbol{m}^{\urcorner}, \boldsymbol{j}^{\urcorner}\right)(j<2)$ which is consistent in that for no $m$ and $A$ are both $A\left({ }^{\ulcorner} m^{\urcorner},{ }^{\ulcorner} \mathbf{0}^{\urcorner}\right)$and $A\left({ }^{\top} m^{\urcorner},{ }^{\ulcorner} 1^{\urcorner}\right)$in the set. Variables such as $P, Q, \ldots$ will range over sets of conditions. The sentences of $\mathscr{L}^{*}$, and the sets of conditions, are supposed to be supplied with a Gödel numbering; a thing and its Gödel number are identified whenever it seems convenient to do so.

Forcing is a relation between sets of conditions and sentences of $\mathscr{L}^{*}$; " $P$ forces $F$ " will be written " $P \Vdash F$." The predicate $P \Vdash F$ is defined by induction on (the number of occurrences of logical constants in) $\boldsymbol{F} . \boldsymbol{F}$ is atomic if it has no logical constants and arithmetical if none of the letters $\boldsymbol{A}$ or $\boldsymbol{A}^{\prime}$ appears in it. $w(e, \boldsymbol{A}, \boldsymbol{P})(n)$ is $e^{A}(n)$ if the latter is determined by $P$; to be precise, $w(e, A, P)(n) \simeq e^{g}(n)$, where $g$ is the partial function $g(x)=j$ if $\left.\boldsymbol{A}\left({ }^{\top} \boldsymbol{x}^{\urcorner},{ }^{\top} \boldsymbol{j}\right\urcorner\right) \in P$ and $g(x)$ undefined otherwise. When it is clear which $\boldsymbol{A}$ is intended, $w(e, A, P)$ will be abbreviated to $e P$; it is safe, for example, to write $A^{\prime}\left({ }^{\top} e P(z)^{\urcorner},{ }^{\top} e P(z+1)^{\urcorner}\right)\left({ }^{\top} e P(z)^{\urcorner}\right.$is of course the $e P(z)$ th formal numeral). In the following definition, clauses 1, 2, 4, 5, and 6 are from Feferman's basic definition [F, §2]. Clause 3 treats the atomic case not present in Feferman's development; it defines $P \Vdash \boldsymbol{A}^{\prime}\left(\ulcorner\boldsymbol{a}\urcorner, \boldsymbol{\zeta}^{\urcorner}\right)$by arithmetical closure conditions suggested by the closure conditions defining $A^{\prime}$. 
Definition 1.

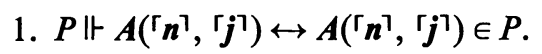

2. If $\boldsymbol{F}$ is an atomic arithmetical sentence, then $P \Vdash F$ if and only if $\boldsymbol{F}$ is true in the natural numbers.

3. $P \Vdash A^{\prime}\left(\Gamma^{\top} \boldsymbol{a}^{\ulcorner} \boldsymbol{r}^{\urcorner}\right)$only as required by:

a. $\left.P \Vdash A^{\prime}\left(\ulcorner 1\urcorner, \Gamma^{\top}\right\urcorner\right)$.

b. $P \Vdash A^{\prime}\left(\left\ulcorner 1^{\top},{ }^{\ulcorner} x^{\top}\right) \rightarrow P \Vdash A^{\prime}\left({ }^{\ulcorner} x^{\top},{ }^{\ulcorner} 2^{x\urcorner}\right)\right.$.

c. If $e P(n)$ is defined, and if

$$
(Q)_{Q \supseteq P}(z)\left(E Q^{\prime}\right)_{Q^{\prime} \supseteq Q} Q^{\prime} \Vdash A^{\prime}\left(\left\ulcorner_{e} Q^{\prime}(z)^{\urcorner},\left\ulcorner_{e} Q^{\prime}(z+1)^{\urcorner}\right),\right.\right.
$$

then $P \Vdash A^{\prime}\left({ }^{\top} e P(n)^{\top},{ }^{\top 3 \cdot 5^{e\urcorner}}\right)$.

(Of course, $Q^{\prime} \Vdash A^{\prime}\left({ }^{\top} e Q^{\prime}(z)^{\top}, \Gamma^{\top} e Q^{\prime}(z+1)^{\urcorner}\right)$cannot hold unless $z$ and $z+1$ are in the domain of e $Q^{\prime}$. This remark applies also in Lemma 1, Lemma 3, and elsewhere.)

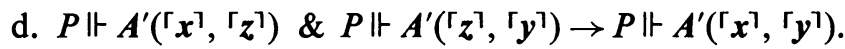

4. $P \Vdash F \vee G \leftrightarrow(P \Vdash F$ or $P \Vdash G)$.

5. $P \Vdash \sim F \leftrightarrow(Q)_{Q \supseteq P} \sim(Q \Vdash F)$.

6. $P \Vdash(E x) F(x) \leftrightarrow(E n)\left(P \Vdash F\left({ }^{\top} n^{\urcorner}\right)\right)$.

There is nothing mysterious about Definition 1, except possibly for clause $3 \mathrm{c}$. The natural thing to attempt for clause $3 \mathrm{c}$ would be something like

$3 c^{\prime}$. If $P \Vdash(x) A^{\prime}\left(\Gamma^{\top A}(x),{ }^{\ulcorner} e^{\rceil A}(x+1)\right)$, then $P \Vdash(x) A^{\prime}\left(\left\ulcorner e^{\top A}(x),{ }^{\top 3} \cdot 5^{e}\right)\right.$.

but that would flagrantly violate our promise to define $P \Vdash F$ by induction on $F$. If one understands how $\mathbb{t}$ ought to behave, then one suspects that enough of the "meaning" of $3 c^{\prime}$ is captured by $3 c$. Note, in particular, that if the conditions on $\boldsymbol{A}$ in $P$ are true of a set $A$, and if $w(e, A, P)(x)$ is defined, then

$$
w(e, A, P)(x)=e P(x)=e^{A}(x) ;
$$

and that past experience suggests $P \Vdash(x) F(x) \leftrightarrow(n)(Q)_{Q \supseteq P}\left(E Q^{\prime}\right)_{Q^{\prime} \supseteq Q} Q^{\prime} \Vdash \boldsymbol{F}\left({ }^{\top} \boldsymbol{n}^{\top}\right)$.

It is convenient first to locate $\Vdash$ as a number-theoretic relation. In general, it ought to be the case that for each proposition $F(X, x)$ the "complexity" of $(\lambda P A x)\left(P \Vdash F\left(A,{ }^{r} x^{\urcorner}\right)\right)$is approximately the minimum, over all sets $A$, of the "complexity" of $(\lambda x) F(A, x)$; and similarly for classes of formulas. Theorem 2 asserts some instances of this principle.

\section{THEOREM 2.}

a. $(\lambda P A a b)\left(P \Vdash A^{\prime}\left(\left\ulcorner^{\urcorner},{ }^{\top} b^{\urcorner}\right)\right)\right.$is $\Pi_{1}^{1}$. (Note that the bound variable $A$ is just a number variable.)

b. $(\lambda P F)(F$ is atomic and $P \Vdash F)$ is $\Pi_{1}^{1}$.

c. $(\lambda P F)(P \Vdash F)$ is hyperarithmetic in $N^{\prime}$; in fact, it is recursive in $\left(N^{\prime}\right)^{(\omega)}$. (Definition. $B^{(0)}=B, B^{(n+1)}=\hat{x}(E y) T_{1}^{B^{(n)}}(x, x, y), B^{(\omega)}=\bigcup_{n} B^{(n)}$.)

Proof. Part a holds because $(\lambda P a b)\left(P \Vdash \boldsymbol{A}^{\prime}\left(\Gamma^{\top} \boldsymbol{a}^{\top},{ }^{\Gamma} \boldsymbol{b}^{\top}\right)\right)$ was defined, uniformly in $\boldsymbol{A}$, by arithmetical closure conditions; part $b$ because the other atomic cases are recursive. For part c, let $r k(F)$ be the total number of negation signs and existential 
quantifiers in $\boldsymbol{F}$. Using part $\mathrm{b}$, it is a routine exercise to show the existence of a recursive function $f$ for which $(\lambda P F)(r k(F)=n \& P \Vdash F)=\{f(n)\}^{\left(N^{\prime}\right)(n)}$, i.e. to show $(\lambda P F)(r k(F)=n \& P \Vdash F)$ to be recursive in $\left(N^{\prime}\right)^{(n)}$ recursively in $n$. Q.E.D.

Sacks suggested the above proof of part $\mathrm{c}$ to us, and pointed out that it applies equally well to Feferman's basic forcing method. Thus the generic sets of $[F, \S 2]$ can be made recursive in $N^{(\omega)}$, essentially the set of true arithmetical sentences, and the hyperarithmetic, but not arithmetically definable, set constructed by Feferman is in fact recursive in $N^{(\omega)}$.

Before the notion of a generic sequence of sets can be defined, another instance is needed of the general lemma on the generation of sets defined by arithmetical closure conditions.

LEMMA 3. There is a partial function $\Psi$ whose graph is $\Pi_{1}^{1}$ and which maps a subset of $Q^{N}$ onto $\left\{\langle P, A, x, y\rangle \mid P \Vdash A^{\prime}\left({ }^{\top} x^{\top},{ }^{\top} y^{\urcorner}\right)\right\}$in such a way that, if $\Psi(a)=\langle P, A, x, y\rangle$ then one of the following holds:

1. $x=1 \& y=2$.

2. $y=2^{x} \&(E b)\left(N^{\prime}(b, a) \& \Psi(b)=\langle P, A, 1, x\rangle\right)$.

3. For some $e$ and $n, y=3 \cdot 5^{e} \& x=e P(n)$

$\&(Q)_{Q \supseteq P}(z)\left(E Q^{\prime}\right)_{Q^{\prime} \supseteq Q}(E b)\left[N^{\prime}(b, a) \& \Psi(b)=\left\langle Q^{\prime}, A, e Q^{\prime}(z), e Q^{\prime}(z+1)\right\rangle\right]$.

4. $(E b)(E c)(E z)\left[N^{\prime}(b, a) \& N^{\prime}(c, a) \& \Psi(b)=\langle P, A, x, z\rangle \& \Psi(c)=\langle P, A, z, y\rangle\right]$.

Proof. Let $Q_{0}, Q_{1}, \ldots$ be a recursive enumeration of all sets of conditions. Let $\Psi$ be the partial function whose graph is the intersection of all solutions $G$ of the following $\Sigma_{1}^{1}$ closure conditions. Then the graph of $\Psi$ is $\Pi_{1}^{1}$. If $a \in Q^{N}$, then fin (a) is the unique natural number $n$ such that $a$ is a notation for $\lambda+n$ with $\lambda=0$ or $\lambda$ a limit ordinal. In the following, $A$ is always $A_{(m)_{0}}$ and $P$ is always $Q_{(m)_{1}}$. Each clause is to be preceded by the condition $a \in Q^{N}$.

1. fin $(a)=2^{m+1} \rightarrow\langle a, P, A, 1,2\rangle \in G$ (i.e. $\left.\Psi(a)=\langle P, A, 1,2\rangle\right)$.

2. [fin $\left.(a)=3^{m+1} \& x=(m)_{2} \&(E b)\left(N^{\prime}(b, a) \&\langle b, P, A, 1, x\rangle \in G\right)\right]$ $\rightarrow\left\langle a, P, A, x, 2^{x}\right\rangle \in G$.

3. $\left\{\right.$ fin $(a)=5^{m+1} \& e=(m)_{2} \& n=(m)_{3} \& x=e P(n)$ $\left.\&(Q)_{Q \supseteq P}(z)\left(E Q^{\prime}\right)_{Q^{\prime} \supseteq Q}(E b)\left[N^{\prime}(b, a) \&\left\langle b, Q^{\prime}, A, e Q^{\prime}(z), e Q^{\prime}(z+1)\right\rangle \in G\right]\right\}$ $\rightarrow\left\langle a, P, A, x, 3 \cdot 5^{e}\right\rangle \in G$.

4. $\left\{\right.$ fin $(a)=7^{m+1} \&(E b)(E c)(E z)\left[N^{\prime}(b, a) \& N^{\prime}(c, a)\right.$

$$
\begin{aligned}
& \left.\left.\qquad\left\langle b, P, A,(m)_{2}, z\right\rangle \in G \&\left\langle c, P, A, z,(m)_{3}\right\rangle \in G\right]\right\} \\
& \rightarrow\left\langle a, P, A,(m)_{2},(m)_{3}\right\rangle \in G .
\end{aligned}
$$

It is clear that the intersection of all solutions for $G$ is in fact the graph of a function, because essentially the same conditions can be regarded as a definition of $\Psi$ by transfinite induction. (That simpler definition of $\Psi$ is not suitable for our purposes because it would not be apparent under that definition that $\Psi$ was $\Pi_{1}^{1}$.) By transfinite induction, one easily verifies that if $\Psi(a)=\langle P, A, x, y\rangle$ then $P \Vdash A^{\prime}\left({ }^{\top} x^{\top},{ }^{\top} y^{\top}\right)$. It remains only to show that the range of $\Psi$ satisfies the closure conditions of 
clause 3 of Definition 1 . Noting that if $b \in Q^{N}$ then there are limit notations exceeding $b$ in $Q^{N}$, one easily verifies conditions a, b, and d. To verify condition c, we make use of Kreisel's lemma [Kr, p. 307], which asserts that if $(x)(E y) P(x, y)$, where $P$ is $\Pi_{1}^{1}$, then there is a hyperarithmetic function $h$ such that $(x) P(x, h(x))$. Recall also that a hyperarithmetic subset of $Q^{N}$ must be bounded in $Q^{N}$, and that the range of a hyperarithmetic function is a hyperarithmetic set.

Suppose then that $e P(n)$ is defined and

$$
(Q)_{Q \supseteq P}(z)\left(E Q^{\prime}\right)_{Q^{\prime} \supseteq Q}(E b)\left[\Psi(b)=\left\langle P, A, e Q^{\prime}(z), e Q^{\prime}(z+1)\right\rangle\right] .
$$

Since the graph of $\Psi$ is $\Pi_{1}^{1}$ there are, by Kreisel's lemma, hyperarithmetic Skolem functions $Q^{\prime}=h(Q, z)$ and $b=k(Q, z)$ for $(*)$. Letting $c \in Q^{N}$ be a bound for the range of $k$, we can replace $(E b)[\Psi(b) \cdots]$ in $(*)$ by $(E b)\left[N^{\prime}(b, c) \& \Psi(b) \cdots\right]$. Then for some $a \in Q^{N}$ not much exceeding $c$, clause 3 of the definition of $\Psi$ applies to yield $\Psi(a)=\left\langle P, A, e P(n), 3 \cdot 5^{e}\right\rangle$. Q.E.D.

If $P \Vdash A^{\prime}\left(\left\ulcorner x^{\urcorner},\left\ulcorner^{\urcorner}\right\urcorner\right)\right.$then $\Psi^{-1}(P, A, x, y)$ is the $N^{\prime}$-least $a \in Q^{N}$ for which $\Psi(a)=$ $\langle P, \boldsymbol{A}, x, y\rangle$, and $\langle P, \boldsymbol{A}, x, y\rangle \prec\langle Q, \boldsymbol{B}, z, w\rangle$ if $N^{\prime}\left(\Psi^{-1}(P, \boldsymbol{A}, x, y), \Psi^{-1}(Q, \boldsymbol{B}\right.$, $z, w)$ ). Since $\Psi$ is $\Pi_{1}^{1}, \prec$ is arithmetical in $N^{\prime}$.

One of the properties which a forcing relation ought to have is that if $P \Vdash F$ and $Q \supseteq P$ then $Q \Vdash F$. Usually it is not a difficult matter to establish this property, but in the present case difficulties are introduced by clause 3 of Definition 1. We are forced to appeal to the function $\Psi$.

THeorem 4. If $P \Vdash F$ and $Q \supseteq P$ then $Q \Vdash F$.

Proof. By induction on $\boldsymbol{F}$, with six cases corresponding to the six clauses of Definition 1. All except Case 3 are easy.

Case 3. $F$ is $A^{\prime}\left({ }^{\top} x^{\urcorner},{ }^{\top} y^{\urcorner}\right)$. This case is handled by induction on

$$
a=a(x, y)=\Psi^{-1}(P, A, x, y) .
$$

There are four subcases, corresponding to the four alternatives of Lemma 3.

Subcase 1. $x=1 \& y=2$, is trivial.

Subcase 2. $y=2^{x} \&(E b)\left(N^{\prime}(b, a) \& \Psi(b)=\langle P, A, 1, x\rangle\right)$. By the induction hypothesis, $\left.Q \Vdash A^{\prime}\left({ }^{\ulcorner} \boldsymbol{1}^{\urcorner},{ }^{\ulcorner} \boldsymbol{x}\right\urcorner\right)$; hence $\left.\left.Q \Vdash \boldsymbol{A}^{\prime}\left({ }^{\top} \boldsymbol{x}\right\urcorner,{ }^{\ulcorner} \boldsymbol{y}\right\urcorner\right)$ according to clause $3 b$ of Definition 1. Subcase 4 is similar.

Subcase 3. $y=3 \cdot 5^{e} \& x=e P(n) \&$

(*) $\quad\left(P^{\prime}\right)_{P^{\prime} \supseteq P}(z)\left(E P^{\prime \prime}\right)_{P^{\prime \prime} \supseteq P^{\prime}}(E b)\left[N^{\prime}(b, a) \& \Psi(b)=\left\langle P^{\prime \prime}, A, e P^{\prime \prime}(z), e P^{\prime \prime}(z+1)\right\rangle\right]$.

Then also $x=e Q(n)$ (because if $g$ extends $f$ and $e^{f}(n)$ is defined, then $e^{g}(n)$ is defined and equal to $e^{f}(n)$ ), and replacing $P$ in (*) by $Q$ clearly results in a true statement. Hence, by Definition $1, Q \Vdash A^{\prime}\left(\left\ulcorner^{\top} \boldsymbol{x}^{\top}, \Gamma^{\urcorner}\right)\right.$. Q.E.D.

The following theorem asserts that the question whether a set of conditions forces a sentence is decided by only those conditions in the set which are "directly relevant." This fact permits great simplifications in the applications of the method. 
TheOREM 5. If $P$ is a set of conditions and $B$ is a fixed $\boldsymbol{A}_{k}$, let

$$
P^{*}=P-\left\{\boldsymbol{B}\left(\left\ulcorner^{\top}\right\urcorner,\ulcorner\boldsymbol{j}\urcorner\right) \mid \text { all } \boldsymbol{n}, \boldsymbol{j}\right\} ;
$$

i.e. $P^{*}$ is the set of conditions in $P$ on sets other than $B$. Let $F$ be any sentence of $L^{*}$ in which neither $\boldsymbol{B}$ nor $\boldsymbol{B}^{\prime}$ occurs. Then $P \Vdash \boldsymbol{F} \leftrightarrow P^{*} \Vdash \boldsymbol{F}$.

Proof. Since $P \supseteq P^{*}$ it suffices to prove $P \Vdash F \rightarrow P^{*} \Vdash F$. The proof is by induction on $\boldsymbol{F}$ and, as usual, only the case " $\boldsymbol{F}$ is $\boldsymbol{A}^{\prime}\left(\left\ulcorner^{\top} \boldsymbol{x},{ }^{\ulcorner} \boldsymbol{y}\right\urcorner\right)$ " (where $\boldsymbol{A}$ is not $\boldsymbol{B}$ ) is not obvious. This case is proved by induction on $a=a(P, x, y)=\Psi^{-1}(P, A, x, y)$, with four subcases, corresponding to the alternatives of Lemma 3. Subcases 1, 2, and 4 are easy.

Subcase 3. $y=3 \cdot 5^{e} \& x=e P(n) \&$

(*) $\quad(Q)_{Q \supseteq P}(z)\left(E Q^{\prime}\right)_{Q^{\prime} \supseteq Q}(E b)\left[N^{\prime}(b, a) \& \Psi(b)=\left\langle Q^{\prime}, A, e Q^{\prime}(z), e Q^{\prime}(z+1)\right\rangle\right]$.

Observe that if $P$ and $Q$ are any sets of conditions, and if $P \supseteq Q^{*}$, then $P^{*}+Q$ is a (consistent) set of conditions.

Now let $Q \supseteq P^{*}$ and fix $z$ arbitrarily. Then $Q^{*}+P$ is a set of conditions, and $Q^{*}+P \supseteq P$. Hence by $(*)$

$$
\left(E Q_{1}\right)_{Q_{1} \supseteq Q^{*+P}}(E b)\left[N^{\prime}(b, a) \& \Psi(b)=\left\langle Q_{1}, A, e Q_{1}(z), e Q_{1}(z+1)\right\rangle\right] .
$$

Then by the induction hypothesis

$$
Q_{1}^{*} \Vdash A^{\prime}\left(\left\ulcorner_{e} Q_{1}^{*}(z)^{\urcorner},\left\ulcorner_{e} Q_{1}^{*}(z+1)^{\urcorner}\right),\right.\right.
$$

since for any $Q, e Q^{*}(x) \simeq e Q(x)$. By the observation, since $Q_{1} \supseteq Q^{*}, Q_{1}^{*}+Q$ is a set of conditions. So

$$
(Q)_{Q \supseteq P^{*}}(z)\left(E Q^{\prime}\right)_{Q^{\prime} \supseteq Q} Q^{\prime} \Vdash A^{\prime}\left(\left\ulcorner^{e} Q^{\prime}(z)^{\urcorner},\left\ulcorner e Q^{\prime}(z+1)^{\urcorner}\right),\right.\right.
$$

namely $Q^{\prime}$ may be taken to be $Q_{1}^{*}+Q$. Thus $P^{*} \Vdash A^{\prime}\left({ }^{\ulcorner} \boldsymbol{x}^{\urcorner},{ }^{\ulcorner} y^{\urcorner}\right)$according to Definition 1. Q.E.D.

1.4. Generic sequences. If $A_{0}, A_{1}, \ldots$ is a sequence of sets then

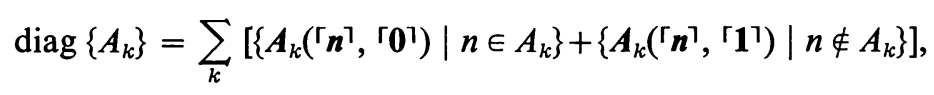

i.e. $\operatorname{diag}\left\{A_{k}\right\}$ is the set of all true conditions on $A_{0}, A_{1}, \ldots$

Definition 2. A sequence $A_{0}, A_{1}, \ldots$ is generic if (1) for every sentence $\boldsymbol{F}$ of $\mathscr{L}^{*}$ there is a $P \subseteq D=\operatorname{diag}\left\{A_{k}\right\}$ such that $P \Vdash F$ or $P \Vdash \sim F$, and (2) if $\boldsymbol{F}$ is $A^{\prime}\left({ }^{\ulcorner} x^{\urcorner},{ }^{\top} y^{\urcorner}\right)$ and $(E P)(P \subseteq D \& P \Vdash F)$ then

$$
(E P)\left(P \subseteq D \& P \Vdash F \&(Q)_{Q \supset P}(\langle P, A, x, y\rangle \prec\langle Q, A, x, y\rangle)\right) .
$$

The usual definition of generic sequence (Cohen [C], Feferman [F]) requires only condition (1). Condition (2) is esthetically displeasing, but it causes no real difficulties and we are unable to establish the basic connection between truth and forcing (Theorem $8, \S 1.5$ ) for sequences not satisfying (2). We see by the following 
theorem that (2) does not interfere with the construction of generic sequences of minimal complexity.

THEOREM 6. There is a generic sequence $A_{0}, A_{1}, \ldots$ such that $\bigcup_{n} A_{n}$ is hyperarithmetic in $N^{\prime}$; in fact $\bigcup_{n} A_{n}$ is recursive in $N^{\prime(\omega)}$.

Proof. Consider the predicate

$$
\begin{aligned}
& \operatorname{Ext}(Q, P, \boldsymbol{F}) \equiv \equiv_{\mathrm{df}} Q \supseteq P \&(Q \Vdash \boldsymbol{F} \vee Q \Vdash \sim \boldsymbol{F}) \&(\boldsymbol{A})(x)(y)\left[\left(\boldsymbol{F} \text { is } \boldsymbol{A}^{\prime}\left({ }^{\ulcorner} \boldsymbol{x}^{\urcorner}, \Gamma^{\ulcorner} \boldsymbol{y}^{\urcorner}\right)\right)\right. \\
&\left.\rightarrow\left(Q^{\prime}\right)_{Q^{\prime} \supset Q}\left(\langle Q, \boldsymbol{A}, x, y\rangle \prec\left\langle Q^{\prime}, \boldsymbol{A}, x, y\right\rangle\right)\right] .
\end{aligned}
$$

Since $\mathbb{t}$ is recursive in $\left(N^{\prime}\right)^{(\omega)}$, and $\prec$ is arithmetical in $N^{\prime}$, Ext is recursive in $\left(N^{\prime}\right)^{(\omega)}$. (The quantifiers in Ext are all just number quantifiers.) It is clear that

$$
(P)(F)(E Q) \operatorname{Ext}(Q, P, F) .
$$

For by clause 5 of Definition 1, there are $Q \supseteq P$ such that $Q \Vdash F$ or $Q \Vdash \sim F$; if $\boldsymbol{F}$ is $\left.\left.\boldsymbol{A}^{\prime}\left({ }^{\top} \boldsymbol{x}\right\urcorner,{ }^{\top} \boldsymbol{y}\right\urcorner\right)$, then choose $Q_{1}$ from this class so as to minimize $\Psi^{-1}\left(Q_{1}, \boldsymbol{A}, \boldsymbol{x}, \boldsymbol{y}\right)$ with respect to the well-ordering $N^{\prime}$.

Hence there is a function $h$ recursive in $\left(N^{\prime}\right)^{(\omega)}$ such that

$$
(P)(F) \operatorname{Ext}(h(P, F), P, F) .
$$

Now let $\boldsymbol{F}_{0}, \boldsymbol{F}_{1}, \ldots$ be a recursive enumeration of the sentences of $\mathscr{L}^{*}$. Set

$$
P(0)=h\left(\varnothing, F_{0}\right), \quad P(n+1)=h\left(P(n), F_{n+1}\right) .
$$

Then $\sum_{n} P(n)$ is the diagram of a generic sequence $A_{0}, A_{1}, \ldots$ Since $x \in A_{k} \leftrightarrow$ $(E n)\left(A_{k}\left({ }^{\ulcorner} \boldsymbol{x}^{\urcorner},{ }^{\ulcorner} \boldsymbol{0}^{\urcorner}\right) \in P(n)\right) \leftrightarrow(n)\left(\boldsymbol{A}_{k}\left(\Gamma^{\top} \boldsymbol{x}^{\urcorner}, \Gamma^{\ulcorner} \mathbf{1}^{\urcorner}\right) \notin P(n)\right)$, with $(\lambda n) P(n)$ recursive in $\left(N^{\prime}\right)^{(\omega)}$, also $\bigcup_{n} A_{n}$ is recursive in $\left(N^{\prime}\right)^{(\omega)}$. Q.E.D.

It is clear that for fixed $Q$ we obtain, by setting $P(0)=h\left(Q, F_{0}\right)$ instead of $h\left(\varnothing, \boldsymbol{F}_{0}\right)$ in the above, a proof of

THEOREM 7. For given $Q$, there is a generic sequence whose diagram includes $Q$. Hence, the generic sequences are dense in $\left(2^{N}\right)^{N}$.

In fact, it is not difficult to prove that the class of generic sequences has a meager (first Baire category) complement in $\left(2^{N}\right)^{N}$. This fact, for Feferman's basic method, has been applied by Addison $\left[\mathrm{A}_{2}\right]$ and Hinman $[\mathrm{H}]$. Similar applications are possible for the present method.

1.5. Forcing and truth. Given a sequence $A_{0}, A_{1}, \ldots$, there is associated with each formal sentence $\boldsymbol{F}$ of $\mathscr{L}^{*}$ a number-theoretic statement $|\boldsymbol{F}|$, obtained from $\boldsymbol{F}$ by replacing each $A_{k}$ by $A_{k}$ and each $A_{k}^{\prime}$ by $A_{k}^{\prime}$, and making the obvious replacements for the other nonlogical constants. We point out that for elementary languages, such as $\mathscr{L}^{*}$, no complicated semantical constructions are necessary for assigning meanings to sentences; nonelementary languages do not have this pleasant property.

In order that a proposed forcing definition be adequate, it is necessary that the following theorem hold. 
THEOREM 8. Let $A_{0}, A_{1}, \ldots$ be a generic sequence, and let $\boldsymbol{F}$ be a sentence of $\mathscr{L}^{*}$. Let $D=\operatorname{diag}\left\{A_{k}\right\}$. Then $|\boldsymbol{F}| \leftrightarrow(E P)(P \subseteq D \& P \Vdash F)$.

As the proof of Theorem 8 is quite complicated, we proceed by more manageable steps. In the end, of course, Theorem 8 will be proved by induction on $\boldsymbol{F}$; the following lemmas will aid in the proof for the case " $\boldsymbol{F}$ is $\left.\boldsymbol{A}^{\prime}\left({ }^{\ulcorner} \boldsymbol{x}\right\urcorner,{ }^{\ulcorner} \boldsymbol{y}\right)$." As indicated by Lemma 9, all the other cases are easy.

Lemma 9. (a) Theorem 8 holds for sentences $\boldsymbol{F}$ in which none of the letters $\boldsymbol{A}^{\prime}$ occur. (b) Hence no set of conditions can force each of a logically inconsistent set of sentences, none of which contains any $\boldsymbol{A}^{\prime}$.

Proof. (a) By induction on $\boldsymbol{F}$, following Feferman [F, Theorem 2.6]. (b) By Theorem 7 and (a). Q.E.D.

Lemma 10. Assume the hypotheses of Theorem 8, and hold $\boldsymbol{A}$ fixed; $\boldsymbol{A}$ is $\boldsymbol{A}_{k}$ if $\boldsymbol{A}$ is $\boldsymbol{A}_{k}$. Set $\left.b(x, y) \simeq\left(N^{\prime}-\min \right)\left\{\Psi^{-1}(Q, A, x, y) \mid Q \subseteq D \& Q \Vdash \boldsymbol{A}^{\prime}\left({ }^{\ulcorner} \boldsymbol{x}\right\urcorner, \Gamma^{\top} \boldsymbol{y}^{\urcorner}\right)\right\}$. Then, if $(E P)\left(P \subseteq D \& P \Vdash A^{\prime}\left({ }^{\top} x^{\urcorner},{ }^{\top} y^{\urcorner}\right)\right)$, one of the following holds:

1. $x=1 \& y=2$.

2. $y=2^{x} \& N^{\prime}(b(1, x), b(x, y))$.

3. $y=3 \cdot 5^{e} \& x=e^{A}(n) \& e^{A}$ is a total function \& $(z) N^{\prime}\left[b\left(e^{A}(z), e^{A}(z+1)\right), b(x, y)\right]$.

4. $(E z)\left[N^{\prime}(b(x, z), b(x, y)) \& N^{\prime}(b(z, y), b(x, y))\right]$.

Proof. By induction on $b=b(x, y)$. There are four cases, corresponding to the four alternatives of Lemma 3; only case 3 is difficult. So let $P$ be such that

$$
\Psi(b)=\langle P, A, x, y\rangle
$$

(so $P \subseteq D$ ), and assume that $y=3 \cdot 5^{e}, x=e P(n)$, and

(*) $(Q)_{Q \supseteq P}(z)\left(E Q^{\prime}\right)_{Q^{\prime} \supseteq Q}(E a)\left[N^{\prime}(a, b) \& \Psi(a)=\left\langle Q^{\prime}, A, e Q^{\prime}(z), e Q^{\prime}(z+1)\right\rangle\right]$.

We first claim that $e^{A}(m)$ is defined for every $m$. For suppose the contrary. By Lemma 9(a) there is, for some $m$, a $Q_{0} \subseteq D$ which forces ( ${ }^{\top} e^{\top A}\left({ }^{\top} \boldsymbol{m}^{\urcorner}\right)$is not defined). Since $P \subseteq D$ and $Q_{0} \subseteq D, Q_{0}+P$ is a set of conditions. By (*) there is a $Q^{\prime} \supseteq Q_{0}+P$ for which $e Q^{\prime}(m)$ is defined. One easily produces a logically inconsistent set of sentences, each forced by $Q^{\prime}$ and none containing any $\boldsymbol{A}^{\prime}$. By Lemma $9(\mathrm{~b})$, this is impossible.

It is evident that $x=e P(n)=e^{A}(n)$.

Now fix $m$ and let $e^{A}(m)=u$ and $e^{A}(m+1)=v$. Choose $Q_{0}$ so that $P \subseteq Q_{0} \subseteq D$ and so that $e Q_{0}(m)=u$ and $e Q_{0}(m+1)=v$. There must be a $Q \subseteq D$ such that

$$
\left.Q \Vdash A^{\prime}\left({ }^{\ulcorner} \boldsymbol{u}^{\urcorner},{ }^{\ulcorner} \boldsymbol{v}\right\urcorner\right) .
$$

For otherwise there is a $Q$, with $Q_{0} \subseteq Q \subseteq D$, such that $\left.Q \Vdash \sim A^{\prime}\left({ }^{\top} \boldsymbol{u}^{\urcorner},{ }^{\ulcorner} \boldsymbol{v}\right\urcorner\right)$. Then by clause 5 of Definition $1,\left(Q^{\prime}\right)_{Q^{\prime} \supseteq Q}(a) \sim\left[\Psi(a)=\left\langle Q^{\prime}, A, u, v\right\rangle\right]$. But for $Q^{\prime} \supseteq Q \supseteq Q_{0}$ we have $e Q^{\prime}(m)=e Q(m)=e Q_{0}(m)=u$, and similarly for $v$. Hence

$$
\left(Q^{\prime}\right)_{Q^{\prime} \supseteq Q}(a) \sim\left[\Psi(a)=\left\langle Q^{\prime}, A, e Q^{\prime}(m), e Q^{\prime}(m+1)\right\rangle\right],
$$

contrary to $(*)$. 
So let $Q \subseteq D$ be such that $Q \Vdash A^{\prime}\left(\Gamma^{\top}{ }^{\top},{ }^{\top} v^{\urcorner}\right)$. We now make essential use of clause 2 of Definition 2. $Q$ may be chosen so that

$$
\left(Q^{\prime}\right)_{Q^{\prime} \supseteq Q}\left(\langle Q, A, u, v\rangle \preccurlyeq\left\langle Q^{\prime}, A, u, v\right\rangle\right) .
$$

Let $Q_{1} \supseteq P+Q+Q_{0}$. Then by (*)

$$
\left(E Q_{2}\right)_{Q_{2} \supseteq Q_{1}}(E a)\left[N^{\prime}(a, b) \& \Psi(a)=\left\langle Q_{2}, A, u, v\right\rangle\right] .
$$

Since $Q_{2} \supseteq Q, b(u, v) \leq_{0}^{N} \Psi^{-1}(Q, A, u, v) \leq_{0}^{N} \Psi^{-1}\left(Q_{2}, A, u, v\right) \leq_{0}^{N} a<_{0}^{N} b=b(x, y)$, which is the third alternative of the present lemma. Q.E.D.

LEMma 11. Assume the hypotheses of Theorem 8. Let $\boldsymbol{G}(\boldsymbol{x})$ be a formula of $\mathscr{L}^{*}$ in which only $x$ is free. If $(m)(E P)\left(P \subseteq D \& P \Vdash G\left({ }^{r} m^{\urcorner}\right)\right)$then

$$
(E P)_{P \subseteq D}(m)(Q)_{Q \supseteq P}\left(E Q^{\prime}\right)_{Q^{\prime} \supseteq Q} Q^{\prime} \Vdash G\left(r^{\top} \boldsymbol{m}^{\top}\right) .
$$

Proof. Look at the sentence $(\boldsymbol{E x}) \sim \boldsymbol{G}(\boldsymbol{x})$. By clauses 5 and 6 of Definition 1 . there is no $P \subseteq D$ which forces $(\boldsymbol{E x}) \sim \boldsymbol{G}(\boldsymbol{x})$. Hence there is a $P \subseteq D$ which forces $\sim(\boldsymbol{E x}) \sim \boldsymbol{G}(\boldsymbol{x})$. Again by clauses 5 and 6 of Definition $1, P$ has the required property. Q.E.D.

Proof of Theorem 8. By induction on $\boldsymbol{F}$. Six cases, corresponding to the clauses of Definition 1. Only case 3 is difficult; for the remainder of the proof, then, let $\boldsymbol{F}$ be $A^{\prime}\left(\left\ulcorner x^{\urcorner}, \Gamma^{\top}\right\urcorner\right)$.

To prove $(E P)(P \subseteq D \& P \Vdash F) \rightarrow|F|$, we use induction on $b(x, y)$, as defined in Lemma 10 . There are four subcases, corresponding to the alternatives of Lemma 10 ; the easy details are left to the reader.

It remains to prove $|\boldsymbol{F}| \rightarrow(E P)(P \subseteq D \& P \Vdash F)$. Let $\Phi$ be a function satisfying Lemma 1. The proof is by induction on $c(x, y) \backsim\left(A^{\prime}-\min \right)\{c \mid \Phi(c)=\langle x, y\rangle\}$; there are four subcases, corresponding to the alternatives of Lemma 1. Only subcase 3 requires explanation. Assume then that $y=3 \cdot 5^{e}, x=e^{A}(n)$, and (as follows immediately from alternative 3 of Lemma 1)

$$
\text { (z) } A^{\prime}\left[c\left(e^{A}(z), e^{A}(z+1)\right), c(x, y)\right]
$$

By the induction hypothesis,

$$
(z)(u)(v)\left[u \neq e^{A}(z) \vee v \neq e^{A}(z+1) \vee(E P)\left(P \subseteq D \& P \Vdash A^{\prime}\left({ }^{\top} u^{\urcorner},{ }^{\ulcorner} v^{\urcorner}\right)\right)\right] .
$$

Applying Lemma 9(a),

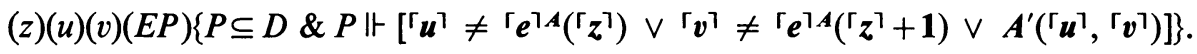
Then by Lemma 11,

(*) $\quad(E P)_{P \subseteq D}(z)(u)(v)\left(Q^{\prime}\right)_{Q^{\prime} \supseteq P}\left(E Q^{\prime \prime}\right)_{Q^{\prime \prime} \supseteq Q^{\prime}}$

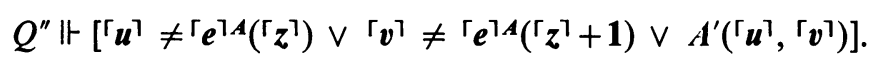

Let $P$ satisfy (*) and also, by Lemma 9(a),

$$
P \Vdash(\ulcorner e\urcorner A \text { is a total function). }
$$


Let $z$ be arbitrary and $Q$ be any extension of $P$. Let $Q^{\prime} \supseteq Q$ so that $u=e Q^{\prime}(z)$ and $v=e Q^{\prime}(z+1)$ are defined; such $Q^{\prime}$ exists by $(* *)$. By $(*)$

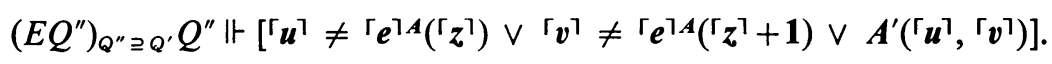

The other possibilities being absurd (since $\left.Q^{\prime \prime} \supseteq Q^{\prime}\right)$, it must be that $Q^{\prime \prime} \Vdash \boldsymbol{A}^{\prime}\left({ }^{\top} \boldsymbol{u}^{\urcorner},{ }^{\ulcorner} \boldsymbol{v}^{\urcorner}\right)$.

It has thus been shown that

$$
(z)(Q)_{Q \supseteq P}\left(E Q^{\prime \prime}\right)_{Q^{\prime \prime} \supseteq Q} Q^{\prime \prime} \Vdash A^{\prime}\left({ }^{\ulcorner} e Q^{\prime \prime}(z)^{\urcorner},\left\ulcorner_{e} Q^{\prime \prime}(z+1)^{\urcorner}\right) ;\right.
$$

hence $P \Vdash A^{\prime}\left({ }^{\top} x^{\urcorner},{ }^{\top} y^{\urcorner}\right)$according to clause $3 c$ of Definition 1. Q.E.D.

1.6. An incomparability result. A set $B$ is said to be arithmetically dependent on a class $\mathscr{K}$ of sets if there is an arithmetical predicate $S\left(X, Y_{0}, \ldots, Y_{n}\right)$ such that, for some $C_{0}, \ldots, C_{n}$ in $\mathscr{K}, B$ is the unique $X$ satisfying $S\left(X, C_{0}, \ldots, C_{n}\right)$. It easy to see that, if $B$ is arithmetically dependent on $\mathscr{K}$, then $B \leq_{h} \bigcup_{0}^{n} C_{k}$ for some $C_{0}, C_{1}, \ldots, C_{n}$ in $\mathscr{K}$. (We write $A \leq_{T} B$ if $A$ is recursive in $B$, and $A \leq_{h} B$ if $A$ is hyperarithmetic in $B$.) The converse is not valid, by Feferman [F, §2]. If $B$ is arithmetical in $\mathscr{K}$ (i.e. in some $C_{0}, \ldots, C_{n}$ in $\mathscr{K}$ ), then $B$ is arithmetically dependent on $\mathscr{K}$; the converse is again false.

In this section we shall prove

THEOREM 12. If $A_{0}, A_{1}, \ldots$ is a generic sequence, then $A_{i}$ is not arithmetically dependent on $\left\{A_{j}^{\prime} \mid j \neq i\right\}$.

Before proving Theorem 12, we state several corollaries.

COROLlaRY 1. There are sets $A$ and B hyperarithmetic in $N^{\prime}$ (in fact, recursive in $\left.\left(N^{\prime}\right)^{(\omega)}\right)$, neither of which is arithmetically dependent on the hyperjumps of the other.

COROLlary 2. There are sets $A$ and B hyperarithmetic in $N^{\prime}$ such that neither set is expressible in either one-function-quantifier form relative to the other set.

COROLlaRY 3. There are sets $A$ and $B$ hypercirithmetic in $N^{\prime}$ whose hyperdegrees are incomparable.

Corollary 4. There is a set $A$ such that $A<_{h} A^{\prime} \equiv_{h} N^{\prime}$ but $A \$_{T} N^{\prime}$.

Corollary 1 follows directly from Theorems 6 and 12. It expresses the strongest "incomparability result" known to us for sets hyperarithmetic in $N^{\prime}$. (Cf. our $\left[\mathrm{T}_{2}\right]$ for stronger incomparability with weaker upper bounds.)

Since in general $C<_{h} N^{\prime} \rightarrow C^{\prime} \equiv_{h} N^{\prime}$, Corollary 1 cannot be strengthened to assert that neither set is hyperarithmetic in the hyperjump of the other.

To prove Corollary 2, note that if $A$ is expressible in either one-functionquantifier form relative to $B$ then $A$ is recursive in, hence arithmetically dependent on, $B^{\prime}$. According to Addison $\left[\mathrm{A}_{1}\right]$, it follows from the axiom of constructibility that of any two sets, one is expressible in both two-function-quantifier forms relative to the other. Hence Corollary 2 is the strongest provable incomparability result expressible just in terms of the analytic hierarchy. 
Corollary 3 is an immediate consequence of Corollary 2. It was first proved by Feferman [F, §3], using forcing applied to a ramified language.

Regarding Corollary 4, we have $A<_{h} A^{\prime} \equiv_{h} N^{\prime}$; but if $A \leq_{T} N^{\prime}$ then $A \leq_{T} N^{\prime}$ $\leq_{T} B^{\prime}$, contrary to Corollary 1 . It is obvious that $C \leq_{T} D \rightarrow C \leq_{h} D$ but not conversely; however the only counterexamples to the converse previously known to us have had $C \equiv_{h} D$.

Proof of Theorem 12. We modify Feferman's [F, §2.12]. Suppose for definiteness that $i=0$. For fixed $n$, define transformations $t$ on sets of conditions and on sentences of $\mathscr{L}^{*}$ by

and

$$
\begin{aligned}
& t(P)=P \text { if for no } j \text { is } A_{0}\left(\Gamma^{\top}{ }^{\top}, \Gamma^{j}\right) \in P
\end{aligned}
$$

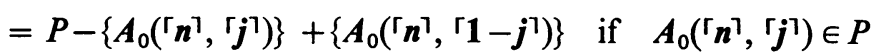

$t(F)$ is the result of replacing each occurrence of $A_{0}(\boldsymbol{u}, v)$ (for any terms $\boldsymbol{u}$ and $v)$ in $F$ by $\left[\left(u={ }^{\top} \boldsymbol{n}^{\top} \& \sim A_{0}(u, v)\right) \vee\left(u \neq{ }^{\top} \boldsymbol{n}^{\top} \& A_{0}(u, v)\right)\right]$.

This $t$ is essentially Feferman's $\tau$ [F, Theorem 2.8]. Following Feferman most of the way, we prove, by induction on $F$, that if the letter $A_{0}^{\prime}$ does not occur in $F$, then $P \Vdash F \leftrightarrow t(P) \Vdash t(F)$. The only case where we must go beyond Feferman's proof is " $F$ is $\left.\boldsymbol{A}^{\prime}\left({ }^{[} \boldsymbol{x}^{\top},{ }^{\top} \boldsymbol{y}\right]\right)$ " (where $\boldsymbol{A}$ is not $\boldsymbol{A}_{0}$ ). In that case $t$ does not change $\boldsymbol{F}$, and by Theorem 5 the possible change which $t$ effects on $P$ is of no consequence.

Now suppose $A_{0}$ to be a solution of $S\left(X, A_{1}^{\prime}, \ldots, A_{m}^{\prime}\right)$. (We show that $A_{0}$ is not the unique solution.) Then $\left|S\left(A_{0}, A_{1}^{\prime}, \ldots, A_{m}^{\prime}\right)\right|$ holds. Let $P \subseteq \operatorname{diag}\left\{A_{k}\right\}$ be such that $P \Vdash S\left(A_{0}, A_{1}^{\prime}, \ldots, A_{m}^{\prime}\right)$. Let $n$ be such that for no $j$ is $\left.A_{0}\left({ }^{[} n^{\top},{ }^{\top} j\right\urcorner\right) \in P$. Use $n$ to define the transformations $t$. Then $t(P)=P$, so $P \Vdash t\left(S\left(A_{0}, A_{1}^{\prime}, \ldots, A_{m}^{\prime}\right)\right)$. Hence $\left|\boldsymbol{t}\left(S\left(A_{0}, A_{1}^{\prime}, \ldots, A_{m}^{\prime}\right)\right)\right|$ holds, i.e. $S\left(C, A_{1}^{\prime}, \ldots, A_{m}^{\prime}\right)$ holds, where $C(x)=A_{0}(x)$ for $x \neq n$ and $C(n)=1-A_{0}(n)$. Q.E.D.

2.1. Applications. In this second part of the paper, the forcing method presented in the first part is applied to the study of the upper semilattice of hyperdegrees. In most cases, the full power of the method is not utilized, the results being stated rather at the level of Corollary 3 to Theorem 12 than at the level of Corollary 1. It seems quite possible that the methods of [F, §3] could be applied at this lower level, but it would be a difficult task.

For each of the problems mentioned at the end of $\$ 1.1$, a modification of the language $\mathscr{L}^{*}$ is devised. The forcing relation previously defined for $\mathscr{L}^{*}$ is then modified slightly to fit the new language. The verification that the usual properties of the forcing relation continue to hold will be omitted since the proofs of $\$ \$ 1.2-1.5$ are easily adapted. The definition of "generic sequence" will remain the same, except that the relation $\prec$ of Definition 2 is implicitly adapted to the new circumstances. Rather more detail may be found in $\left[T_{3}\right]$.

2.2. A hyperdegree incomparable with a given nonzero hyperdegree. $\mathscr{L}$ remains the language of elementary number theory. Let $B$ be a given nonhyperarithmetic 
set. We are to find a set hyperarithmetically incomparable with $B . \mathscr{L}(B)$ is obtained from $\mathscr{L}$ by adjoining binary predicate letters $B$ representing (the representing predicate of the representing function of) the set $B$, and $B^{\prime}$ representing the hyperjump $B^{\prime}$ of $B . \mathscr{L}^{*}(B)$ is obtained from $\mathscr{L}(B)$ by adjoining a single pair $A, A^{\prime}$ of "generic" binary predicate letters. (We construct a single generic set rather than an infinite sequence just for simplicity of notation.) A sentence of $\mathscr{L}^{*}(B)$ is said to be atomic if none of the logical constants appears in it, and arithmetical if it is a sentence of $\mathscr{L}(B)$. Each arithmetical sentence has an unambiguous meaning at the outset; a meaning is assigned to an arbitrary sentence simply by choosing a set $A$ as the interpretation of $\boldsymbol{A}$. A set of conditions is a finite consistent set of sentences of the form $A\left({ }^{\top} n^{\urcorner},{ }^{\top} j^{\top}\right)(j<2)$. The forcing relation for $\mathscr{L}^{*}(B)$ is defined by Definition 1 (with the new meaning of "arithmetical" and with $\boldsymbol{A}$ now being $\boldsymbol{A}$ and not an arbitrary $\boldsymbol{A}_{i}$ ).

By an easy modification of $\S 1.3$, the forcing relation is hyperarithmetic in $B^{\prime}$. The notion of generic set is defined as in $\S 1.3$. Then there is a generic set $A \leq_{h} B^{\prime}$.

THEOREM 13. If $A$ is generic, then $A$ and $B$ have incomparable hyperdegrees; in fact hyp $(A) \cap$ hyp $(B)=\mathbf{0}$.

[hyp $(A)$ is the hyperdegree of $A$; ${ }^{\top} 0^{\urcorner}=$hyp $(N)$; if ${ }^{\top} h^{\top}$ and ${ }^{\ulcorner} k^{\urcorner}$are hyperdegrees then $\boldsymbol{h} \cup \boldsymbol{k}$ is their least upper bound, $\boldsymbol{h} \cap \boldsymbol{k}$ is their greatest lower bound, if it exists, and $\boldsymbol{h}^{\prime}$ is the hyperjump of $\boldsymbol{h}$.]

Proof. The proof of Theorem 12 is easily modified to show that $A$ is not arithmetically definable from $B^{\prime}$; it follows that $A$ is not hyperarithmetic, so we need only show hyp $(A) \cap$ hyp $(B)=\mathbf{0}$.

Suppose first that $S$ is a set $\Pi_{1}^{1}$ in $A$ and in $B$. Then by [Sp, $\left.\S 2,3\right]$ there are primitive recursive functions $f$ and $g$ such that

$$
(x)\left\{x \in S \leftrightarrow A^{\prime}\left(f(x), 2^{f(x)}\right) \leftrightarrow B^{\prime}\left(g(x), 2^{g(x)}\right)\right\} .
$$

By the fundamental property of generic sets (Theorem 8 ) there is a

$$
P \subseteq \operatorname{diag}(A)=\left\{A\left({ }^{\top} \boldsymbol{n}^{\urcorner},{ }^{\ulcorner} \mathbf{0}^{\urcorner}\right) \mid n \in A\right\}+\left\{A\left({ }^{\ulcorner} \boldsymbol{n}^{\urcorner},{ }^{\ulcorner} \mathbf{1}^{\urcorner}\right) \mid n \notin A\right\}
$$

such that

$$
P \Vdash(x)\left[A^{\prime}\left(f(x), 2^{f(x)}\right) \leftrightarrow B^{\prime}\left(g(x), 2^{g(x)}\right)\right]
$$

(The "sentence" above is of course only an abbreviation for a proper sentence of $\mathscr{L}^{*}(B)$.) We claim that

$$
(x)\left[x \in S \leftrightarrow ( E Q ) _ { Q \supseteq P } Q \Vdash A ^ { \prime } \left(\left\ulcorner f(x)^{\urcorner},\left\ulcorner^{\ulcorner}{ }^{f(x)} 7\right)\right] .\right.\right.
$$

Since the forcing relation restricted to sentences of the form $A^{\prime}\left({ }^{\top} x^{\urcorner}, \Gamma^{\top} y^{\urcorner}\right)$is $\Pi_{1}^{1}$ (as in Theorem 2), and remembering that $Q$ is just a number variable, (3) expresses $S$ in $\Pi_{1}^{1}$ form.

Now one half of the claim is trivial, for if $x \in S$ then $A^{\prime}\left(\left[f(x)^{\top},\left\ulcorner^{f(x)\urcorner}\right)\right.\right.$ is true, so there is a $Q$ with $P \subseteq Q \subseteq \operatorname{diag}(A)$ such that $Q \Vdash A^{\prime}\left(\left[f(x)^{\urcorner},{ }^{\ulcorner} 2^{f(x)\urcorner}\right)\right.$. To prove the 
other half, suppose that $Q \supseteq P$ and $Q \Vdash A^{\prime}\left(\left[f(x){ }^{1},{ }^{\ulcorner} 2^{f(x) 1}\right)\right.$. By (2), there is a $Q_{1} \supseteq Q$ such that

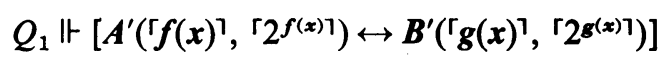

and then because of the assumption on $Q$, there is a $Q_{2} \supseteq Q_{1}$ such that

$$
Q_{2} \Vdash \boldsymbol{B}^{\prime}\left(r^{g}(\boldsymbol{x})^{\urcorner}, r^{\left.\boldsymbol{g}^{(x)}\right)}\right) .
$$

But the latter sentence is arithmetical (as the term was defined for $\mathscr{L}^{*}(B)$ ) and can be forced only if it is true. By (1) it follows that $x \in S$.

Thus any set $\Pi_{1}^{1}$ in $A$ and in $B$ is $\Pi_{1}^{1}$ absolutely. The same holds with $\Sigma_{1}^{1}$ in place of $\Pi_{1}^{1}$ (consider the complement of $S$ ), so that any set hyperarithmetic in $A$ and in $B$ is hyperarithmetic. Q.E.D.

Corollary 1. If $0<\boldsymbol{h}$, then there is a $\boldsymbol{k}$ with $\mathbf{0}<\boldsymbol{k}<\boldsymbol{h}^{\prime}, \boldsymbol{h} \mid \boldsymbol{k}$, and $\boldsymbol{h} \cap \boldsymbol{k}=\mathbf{0}$.

It is not difficult to prove that the class of nongeneric sets is meager (first Baire category) in $2^{N}$ (with the usual product topology). Hence we have also

COROLLARY 2. If B is not hyperarithmetic, then the class of sets hyperarithmetically comparable with $B$ is meager.

Since $\omega_{1}^{B}>\omega_{1}$ only if ${ }^{r} 0^{\prime \prime} \leq$ hyp $(B)$, it follows that

COROLLARY 3. $\omega_{1}^{B}=\omega_{1}$, except for a meager class of sets $B$.

2.3. Countable embeddings. Let $P$ be any set and $\leq$ be a partial ordering of $P$. An embedding of $P$ in the hyperdegrees is a mapping $\phi: P \rightarrow 2^{N}$ such that

$$
\phi(a) \leq_{h} \phi(b) \leftrightarrow a \leq b .
$$

Denote least upper bounds and greatest lower bounds in $P$ by $\cup$ and $\cap$, respectively. An embedding $\phi$ is said to be $\cup$-preserving [ $\cap$-preserving] if whenever $a \cup b$ exists $\phi(a \cup b) \equiv_{h} \phi(a) \cup \phi(b)$ [whenever $a \cap b$ exists hyp $(\phi(a)) \cap$ hyp $(\phi(b))$ exists and is equal to hyp $(\phi(a \cap b))]$. Finally, $\phi$ is lattice-preserving if it is both $\cup$-preserving and $\cap$-preserving.

THEOREM 14. There is a lattice-preserving embedding of the lattice $\operatorname{Rec}$ of recursive sets under inclusion in the hyperdegrees less than $0^{\prime}$.

Proof. Let $\mathscr{L}^{*}(\mathrm{Rec})$ be the language of elementary arithmetic augmented by binary predicates $A_{R}$ and $A_{R}^{\prime}$ for all $R \in \operatorname{Rec} . A_{R}$ is intended to represent

$$
\bigcup\left\{A_{k} \mid k \in R\right\}=\left\{p_{k}^{x+1} \mid k \in R \wedge x \in A_{k}\right\}
$$

where the $A_{k}$ are unspecified sets, and $A_{R}^{\prime}$ is intended to represent the hyperjump of $A_{R}$. The definition of $P \Vdash F$ is altered accordingly, as follows. A set of conditions remains a finite, consistent set of sentences (but not sentences of $\mathscr{L}^{*}(\operatorname{Rec})$ ) of the form $\left.\left.\boldsymbol{A}_{k}\left(\boldsymbol{r}^{\top}\right\urcorner, \Gamma^{\top}\right\urcorner\right)(j<2)$. 


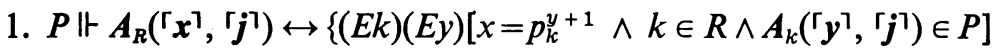

$$
\vee\left[j=1 \wedge \sim(E k)(E y)\left[x=p_{k}^{y+1} \wedge k \in R\right]\right\} .
$$

3. [As 3 of Definition 1, with $\boldsymbol{A}^{\prime}$ replaced by $\boldsymbol{A}_{R}^{\prime}$ and $e P$ taken as an abbreviation for $e P R$, which is $\{e\}^{A_{R}}$ insofar as the latter is determined by $P$.]

\section{$2,4,5,6$. [As in Definition 1.]}

Using a suitable arithmetical enumeration of the recursive sets, a Gödel numbering of the sentences of $\mathscr{L}^{*}(\operatorname{Rec})$ can be established; thus $\Vdash$ for $\mathscr{L}^{*}(\mathrm{Rec})$ becomes a number-theoretic relation. As in $\S 1.3$, It is hyperarithmetic in $N^{\prime}$, and there is a generic sequence $A_{0}, A_{1}, \ldots$ of sets, all hyperarithmetic in $N^{\prime}$. Define $\phi: \operatorname{Rec} \rightarrow 2^{N}$ by $\phi(R)=A_{R}=\bigcup\left\{A_{n} \mid n \in R\right\}$. It is claimed that $\phi$ is a lattice-preserving embedding.

Plainly, $R \subseteq S \rightarrow \phi(R) \leq_{h} \phi(S)$. Conversely, if $R \nsubseteq S$, let $y \in R-S$. The methods of $\$ 1.6$ show that $A_{y} \Varangle_{h} A_{S}$. But $A_{y} \leq_{h} A_{R}$, so it follows that $A_{R} \hbar_{h} A_{S}$. It is also clear that $\phi$ is $\cup$-preserving. To see that $\phi$ is $\cap$-preserving, note first that

$$
\text { hyp }(\phi(R \cap S))
$$

is a lower bound for hyp $(\phi(R))$ and hyp $(\phi(S))$ because of the order-preserving property of $\phi$ established above. It remains only to prove that whenever $B \leq_{h} A_{R}$ and $B \leq_{h} A_{S}$ then $B \leq_{h} A_{R \cap S}$.

In fact, a stronger statement holds. Suppose $B$ is $\Pi_{1}^{1}$ in $A_{R}$ and $\Sigma_{1}^{1}$ in $A_{S}$. Then there are recursive $f, g$ such that

$$
(x)\left\{x \in B \leftrightarrow A_{R}^{\prime}\left(f(x), 2^{f(x)}\right) \leftrightarrow \sim A_{S}^{\prime}\left(g(x), 2^{g(x)}\right)\right\},
$$

and hence there is a $P \subseteq \operatorname{diag}\left\{A_{n}\right\}$ such that

$$
P \Vdash(x)\left[A_{R}^{\prime}\left(f(x), 2^{f(x)}\right) \leftrightarrow \sim A_{S}^{\prime}\left(g(x), 2^{g(x)}\right)\right] .
$$

Let $\operatorname{Agr}(Q)$ mean that $Q$ agrees with $\operatorname{diag}\left\{A_{n}\right\}$ for indices in $R \cap S$, i.e.,

$$
(x)(j)(n)\left[n \in R \cap S \wedge A_{n}\left(\left\ulcorner x^{\urcorner},\left\ulcorner^{\top}\right\urcorner\right) \in Q \rightarrow A_{n}(x, j)\right] .\right.
$$

Then we shall see that

$$
\begin{aligned}
& x \in B \leftrightarrow(E Q)_{Q \supseteq P}\left[\operatorname{Agr}(Q) \wedge Q \Vdash A_{R}^{\prime}\left(\ulcorner f(x)\urcorner,\left\ulcorner 2^{f(x)\urcorner}\right)\right]\right. \\
& x \notin B \leftrightarrow(E Q)_{Q \supseteq P}\left[\operatorname{Agr}(Q) \wedge Q \Vdash A_{S}^{\prime}\left(\left\ulcorner g(x){ }^{\urcorner},{ }^{\ulcorner} 2^{(x)} 7\right)\right] .\right.
\end{aligned}
$$

The above express $B$ and its complement in $\Pi_{1}^{1}$ form relative to $A_{R \cap S}$ (for $(E Q)$ is a number quantifier, Agr is arithmetical in $A_{R \cap S}$, and $\Vdash$ is $\Pi_{1}^{1}$ absolutely); thus it will follow that $B \leq_{h} A_{R \cap S}$.

The implications from left to right in (2) and (3) are trivial, for $Q$ can be taken $\subseteq \operatorname{diag}\left\{A_{n}\right\}$. Suppose then that $Q_{1} \supseteq P, \operatorname{Agr}\left(Q_{1}\right)$, and $Q_{1} \Vdash A_{R}^{\prime}\left(\ulcorner f(x)\urcorner,\left\ulcorner^{\ulcorner} f^{f(x)}\right\urcorner\right)$. By the analogue for the present language of Theorem 5, we may assume that $Q_{1}-P$ mentions $A_{n}$ only for $n \in R$. Now suppose also that $x \notin B$. Then there is a $Q_{2} \supseteq P$ such that $\operatorname{Agr}\left(Q_{2}\right)$ and $Q_{2} \Vdash \boldsymbol{A}_{S}^{\prime}\left({ }^{\prime} \boldsymbol{g}(\boldsymbol{x})^{\top},{ }^{\ulcorner} 2^{(x) 7}\right)$. Again, it is safe to assume that 
$Q_{2}-P$ mentions $A_{n}$ only for $n \in S$. The requirements " $\operatorname{Agr}\left(Q_{i}\right)$ " " $Q_{1}-P$ mentions only $\boldsymbol{A}_{R}$ " " $Q_{2}-P$ mentions only $\boldsymbol{A}_{S}$," and " $Q_{i} \supseteq P$ " together assure that $Q_{1}+Q_{2}$ is a (consistent) set of conditions. But $Q_{1}+Q_{2}$ forces both $\boldsymbol{A}_{R}^{\prime}\left({ }^{[} \boldsymbol{f}(\boldsymbol{x})^{\top},\left\ulcorner^{\left.{ }^{f(x)}\right\urcorner}\right)\right.$ and

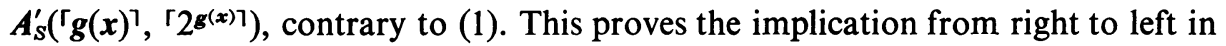
(2), and (3) is treated similarly. Q.E.D.

We shall make use of the following well-known proposition (for which we are unable to find a reference), which was called to our attention by Professor Anil Nerode.

Proposition 1. Let B be a countable Boolean algebra of subsets of $N$ which contains every finite set and which contains, with each infinite set $M$, two infinite disjoint sets whose union is $M$. Then $B$ is isomorphic to the Boolean algebra of recursive sets.

As a corollary of Proposition 1, we have

Proposition 2. If $P$ is any countable partially ordered set then there is a U-preserving [n-preserving] embedding of $P$ in the lattice $R e c$ of recursive sets.

Proof. Assume, without loss of generality, that $P \subseteq N$; let $\leq_{1}$ be the partial ordering of $P$. Define $J: P \rightarrow 2^{N}$ by $J(a)=\left\{m \mid a \neq_{1} m\right\}$. Then $J(a \cup b)=J(a)+J(b)$ whenever $a \cup b$ exists. $J[P]$ can easily be extended to a Boolean algebra $B$ satisfying Proposition 1. The $\cup$-preserving embedding of $P$ in Rec consists of $J$, followed by an isomorphism $B \rightarrow$ Rec. To obtain an $\cap$-preserving embedding, replace $J(a)$ by $K(a)=\left\{m \mid m \leq_{1} a\right\}$. Q.E.D.

COROLLARY 1. If $P$ is a countable partially ordered set then there is a $\cup$-preserving [n-preserving] embedding of $P$ in the hyperdegrees less than $\mathbf{0}^{\prime}$.

It is not difficult to prove that the lattice-preserving embedding of Theorem 14 is also a lattice-preserving embedding in the degrees. (Details appear in $\left[\mathrm{T}_{3}\right]$.) Hence we have also

CoRollary 2. There is a lattice-preserving embedding of $\operatorname{Rec}$ in the degrees. If $P$ is a countable partially ordered set, there is a $\cup$-preserving [ $\cap$-preserving] embedding of $P$ in the degrees.

2.4. Uncountable embeddings. We turn now to the problem of embedding a partially ordered set $P$, of cardinality at most that of the continuum, such that each element of $P$ has only finitely many predecessors.

THEOREM 15. Let Fin be the lattice of finite subsets of $2^{N}$. There is a latticepreserving embedding of Fin in the hyperdegrees.

Proof. Consider the languages $\mathscr{L}^{*}(n)$, which are like $\mathscr{L}^{*}(\mathrm{Rec})$ except that pairs $A_{R}, A_{R}^{\prime}$ are adjoined only for $R \subseteq\{0,1, \ldots, n-1\}$. A set of conditions is a set of sentences $\left.\boldsymbol{A}_{k}\left(\boldsymbol{}^{\mathrm{r}} \boldsymbol{m}^{\top},{ }^{\mathrm{j}} \mathrm{j}\right]\right)$ for $j<2, k<n$. A generic sequence for $\mathscr{L}^{*}(n)$ is then a finite 
sequence $A_{0}, \ldots, A_{n-1}$ of sets. By a proof similar to that of Theorem 14, if $A_{0}, \ldots$, $A_{n-1}$ is generic for $\mathscr{L}^{*}(n)$, then $\phi$ is a lattice-preserving embedding in the hyperdegrees of the lattice of subsets of $\{0, \ldots, n-1\}$, where $\phi(R)=A_{R}=\bigcup\left\{A_{m} \mid m \in R\right\}$. Hence, to prove the present theorem it suffices to construct sets $A_{\alpha}\left(\alpha \in 2^{N}\right)$ such that whenever $\alpha_{0}, \ldots, \alpha_{n-1}$ are all distinct then $A_{\alpha_{0}}, \ldots, A_{\alpha_{n-1}}$ is generic for $\mathscr{L}^{*}(n)$; the map $\psi$ : Fin $\rightarrow 2^{N}$ defined by $\psi\left(\left\{\alpha_{0}, \ldots, \alpha_{m}\right\}\right)=\bigcup\left\{A_{\alpha_{j}} \mid j \leq m\right\}$ is the desired embedding. [To make $\psi$ well-defined, select any linear ordering of $2^{N}$ and order finite subsets according to the induced order.]

We omit most of the formal details of the construction of the sets $A_{\alpha}$ (they appear in $\left[\mathrm{T}_{3}\right]$ ) and present an informal outline. One constructs generic sequences by considering in turn each of the (countably many) sentences of the language involved; at the time the sentence $\boldsymbol{H}$ is considered, one makes the sequence "generic with respect to $\boldsymbol{H}$."

The sets $A_{\alpha}$ will be approximated by partial functions $A_{\tilde{\alpha}(n)}$ with finite domains. The notation is intended to convey that $A_{\tilde{\alpha}(n)}$ depends only on $\bar{\alpha}(n)$, and not really on $\alpha$. At stage $r$ we shall, in several steps, extend the partial functions $A_{s}$ (for sequence numbers $s$, of length $r$, representing sequences of 0 's and 1's) to partial functions $A_{t}(l h(t)=r+1)$, so that $A_{t}$ extends $A_{s}$ whenever $t$ extends $s$. We consider, at stage $r$, a particular sentence $H$ of a particular language $\mathscr{L}^{*}(n)$. We attempt at stage $r$ to make $A_{\alpha_{0}}, \ldots, A_{\alpha_{n-1}}$ "generic with respect to $\boldsymbol{H}$," for all sequences $\alpha_{0}, \ldots, \alpha_{n-1}$ of distinct members of $2^{N}$. In this attempt we shall be only partly successful, because at stage $r$ we can distinguish between $A_{\alpha}$ and $A_{\beta}$ only if $\bar{\alpha}(r+1) \neq \bar{\beta}(r+1)$. We therefore attack $\boldsymbol{H}$ at infinitely many stages so that eventually we distinguish among any fixed distinct $\alpha_{0}, \ldots, \alpha_{n-1}$. Now at stage $r$ there are finitely many ways of associating the sequence numbers of length $r+1$ with the $n$ numerical subscripts of the adjoined pairs $A_{k}, A_{k}^{\prime}$ of $\mathscr{L}^{*}(n)$. Each step of stage $r$ corresponds to one such association. Let $A_{\tilde{\alpha}(r+1)}^{0}=A_{\tilde{\alpha}(r)}$. The first association determines a set of conditions for $\mathscr{L}^{*}(n)$, which is extended as demanded by $\boldsymbol{H}$; the extension in turn determines new partial functions $A_{\tilde{\alpha}(r+1)}^{1}$ extending $A_{\tilde{\alpha}(r+1)}^{0}$. Proceed thus through all the steps, and let $A_{\tilde{\alpha}(r+1)}$ be the final result. The required genericity is evident. Q.E.D.

COROLlaRY 1. Let $P$ be a partially ordered set of cardinality at most that of the continuum, such that each element of $P$ has only finitely many predecessors. Then there is an $\cap$-preserving embedding of $P$ in the hyperdegrees.

COROLlaRY 2. There is a lattice-preserving embedding of Fin in the degrees; if $P$ satisfies the hypotheses of Corollary 1 , then there is an $\cap$-preserving embedding of $P$ in the degrees.

Proofs. Suppose, without loss of generality, that $P \subseteq 2^{N}$; let $\leq_{1}$ be the ordering of $P$. Then $K(a)=\left\{b \mid b \leq_{1} a\right\}$ is an $\cap$-preserving embedding of $P$ in Fin. Follow $K$ with a lattice-preserving embedding of Fin in the hyperdegrees, proving Corollary 1. Corollary 2 is proved similarly, after noting that the embedding of Fin in the 
hyperdegrees given by Theorem 15 is also a lattice-preserving embedding in the degrees. Again, the details may be found in $\left[T_{3}\right]$. Q.E.D.

In [Sa, \$\$2-4] Sacks proved several theorems concerning embeddability of partial orderings in the degrees. Some of these are extended to $\cup$-preserving, $\cap$-preserving, or lattice-preserving embeddability by our Corollaries 2 to Theorems 14 and 15.

On the other hand, we have not been able to prove embeddability in the hyperdegrees for all the partial orderings which Sacks proved embeddable in the degrees. It should be noted that Theorems 1 and 3 of [Sa, §3] are not true of the hyperdegrees, because their truth would imply that no infinite ascending sequence of hyperdegrees has a least upper bound. Thus it will not be easy to adapt Sacks' arguments to the hyperdegrees.

2.5. Complete hyperdegrees. The hyperdegree $\boldsymbol{h}$ is said to be complete if $\boldsymbol{h}=\boldsymbol{k}^{\prime}$ for some hyperdegree $\left.{ }^{\top} \boldsymbol{k}\right\urcorner$. Evidently, if $\boldsymbol{h}$ is complete then $\boldsymbol{h} \geq \boldsymbol{0}^{\prime}$. In this section we shall prove the converse. For the remainder of the paper, we shall work with the language $\mathscr{L}_{1}^{*}$, obtained from $\mathscr{L}$ by adjoining just one pair $\boldsymbol{A}, \boldsymbol{A}^{\prime}$ of "generic" constants.

Lemma 16. If $A$ is generic, then $A^{\prime} \equiv_{T} A \cup N^{\prime}$.

Proof. It is well known that $N^{\prime} \leq_{T} A^{\prime}$ and $A \leq_{T} A^{\prime}$.

If $A$ is generic, then

$$
\begin{aligned}
A^{\prime}(x, y) & \leftrightarrow(E P)\left[P \subseteq \operatorname{diag}(A) \wedge P \Vdash A^{\prime}\left(\left\ulcorner x^{\urcorner}, \Gamma^{\top} y^{\urcorner}\right)\right]\right. \\
& \leftrightarrow(P)\left[P \subseteq \operatorname { d i a g } ( A ) \rightarrow \sim P \Vdash \sim A ^ { \prime } \left(\left\ulcorner x^{\urcorner},\left\ulcorner y^{\urcorner}\right)\right] .\right.\right.
\end{aligned}
$$

Now $(\lambda P x y) P \Vdash A^{\prime}\left(\left\ulcorner^{\top}\right\urcorner,{ }^{\ulcorner} \boldsymbol{y}^{\urcorner}\right)$is $\Pi_{1}^{1}$ and $(\lambda P x y) P \Vdash \sim \boldsymbol{A}^{\prime}\left(\left\ulcorner^{\top} \boldsymbol{x}^{\urcorner},\left\ulcorner^{\top} \boldsymbol{y}^{\urcorner}\right)\right.\right.$is $\Sigma_{1}^{1}$; thus both are recursive in $N^{\prime}$. Hence the above express $A^{\prime}$ in both one-number-quantifier forms relative to $A, N^{\prime}$. Q.E.D.

Corollary. If $A$ is generic, then $N^{\prime} \ddagger_{h} A$.

THEOREM 17. There is a function $h \leq_{h} N^{\prime}$ such that $(B)(E A)[(A$ is generic $) \wedge$ $\left.\left(A \leq_{h} N^{\prime} \cup B\right) \wedge(n)(n \in B \leftrightarrow h(n) \in A)\right]$.

Proof. Let Ext $(Q, P, F)$ be the predicate introduced in the proof of Theorem 6; Ext $(Q, P, F)$ means, in the language of $\S 2.4$, that $Q$ is an extension of $P$ which makes $A$ generic with respect to the sentence $F$. It was shown that Ext $\leq_{h} N^{\prime}$ and that $(P)(F)(E Q)$ Ext $(Q, P, F)$. Three functions $P_{s}(n), Q_{s}(n)$, and $h(n)$ are to be defined simultaneously by induction on $n$, uniformly for sequence numbers $s$ representing sequences of 0 's and 1's with $\ln (s) \geq n+1 . F_{0}, F_{1}, \ldots$ is a recursive enumeration of all the sentences of $\mathscr{L}_{1}^{*}$, and $\mu P$ means the least $P$ in some recursive well-ordering of all sets of conditions.

$$
\begin{aligned}
& P_{s}(0)=(\mu P) \operatorname{Ext}\left(P, \varnothing, \boldsymbol{F}_{0}\right) . \\
& h(n)=1+\max \left\{\boldsymbol{m} \mid(E s)(E j)\left[\operatorname{lh}(s)=n+1 \wedge\left(A\left({ }^{\ulcorner} \boldsymbol{m}^{\urcorner},\left\ulcorner^{\top} \boldsymbol{j}^{\urcorner}\right) \in P_{s}(n)\right)\right]\right\} .\right. \\
& Q_{s}(n)=P_{s}(n)+\left\{\boldsymbol{A}\left({ }^{\ulcorner} h(n)^{\urcorner},\left\ulcorner(s)_{n}{ }^{\urcorner}\right)\right\} .\right. \\
& P_{s}(n+1)=(\mu P) \operatorname{Ext}\left(P, Q_{s}(n), \boldsymbol{F}_{n+1}\right) .
\end{aligned}
$$


Then $h$ is recursive in Ext, hence hyperarithmetic in $N^{\prime}$. For any fixed $B$, let $A$ be the set whose diagram is $\sum_{n} P_{\bar{B}(n+1)}(n)$. Then $h$ and $A$ meet the requirements of the theorem. Q.E.D.

Corollary 1. If $B \geq_{h} N^{\prime}$, then there is a generic set $A$ such that $B \equiv_{h} A \cup N^{\prime}$ $\equiv_{h} A^{\prime}$.

COROLlaRY 2. There are sets $A$ such that $\omega_{1}^{A}=\omega_{1}$ but $\omega_{1}^{A^{\prime}}$ is arbitrarily large.

\subsection{Two hyperdegrees whose least upper bound is $\mathbf{0}^{\prime}$.}

THEOREM 18. There are generic sets $A_{0}, A_{1}$ such that $A_{0} \cup A_{1} \equiv_{h} N^{\prime}$.

Proof. We approximate the sets $A_{i}$ by partial representing functions $A_{i}^{n} ; A_{i}^{n+1}$ is always an extension of $A_{i}^{n} . A_{i}^{0}$ is the empty function. At stage $2 n$, proceed as follows:

Set $Q_{0}=\left\{\boldsymbol{A}\left({ }^{\ulcorner} \boldsymbol{x}^{\top}, \Gamma^{\top} \boldsymbol{j}\right) \mid A_{0}^{n}(x)=j\right\}$.

$$
\begin{aligned}
& P=(\mu Q) \operatorname{Ext}\left(Q, Q_{0}, F_{n}\right) . \\
& A_{0}^{n+1}(x)=j \leftrightarrow A\left({ }^{\top} x^{\urcorner}, \Gamma^{\urcorner}\right) \in P . \\
& A_{1}^{n+1}(x)=j \leftrightarrow\left\{\left(x \in N^{\prime} \wedge A_{0}^{n+1}(x)=j\right) \vee\left(x \notin N^{\prime} \wedge A_{0}^{n+1}(x)=1-j\right)\right\} .
\end{aligned}
$$

At stage $2 n+1$, interchange the roles of $A_{0}$ and $A_{1}$. Then each $A_{i}$ is generic; and $A_{i}$ is recursive in Ext, $N^{\prime}$, hence hyperarithmetic in $N^{\prime}$. Since

$$
x \in N^{\prime} \leftrightarrow\left[x \in A_{0} \leftrightarrow x \in A_{1}\right], \quad N^{\prime} \leq_{T} A_{0} \cup A_{1} . \quad \text { Q.E.D. }
$$

COROLlARY 1. There are hyperdegrees $\boldsymbol{h}_{0}, \boldsymbol{h}_{1}$ such that $\boldsymbol{h}_{0}<\boldsymbol{0}^{\prime}, \boldsymbol{h}_{1}<\boldsymbol{0}^{\prime}, \boldsymbol{h}_{0} \mid \boldsymbol{h}_{1}$, and $\boldsymbol{h}_{0} \cup \boldsymbol{h}_{1}=\mathbf{0}^{\prime}$.

Proof. By Theorem 18 and the corollary to Lemma 16. Q.E.D.

COROLlaRY 2. If $\boldsymbol{h} \geq \mathbf{0}^{\prime}$, then $\boldsymbol{h}$ is the least upper bound of its proper predecessors.

Proof. By Corollary 1 and Theorem $17, \boldsymbol{h}=$ hyp $\left(B^{\prime}\right)$ for some $B$. By relativizing the proof of Theorem 18, obtain sets $A_{0}, A_{1}<_{h} B^{\prime}$ such that $A_{0} \cup A_{1} \equiv_{h} B^{\prime}$.

We do not know whether there are incomparable hyperdegrees $\boldsymbol{h}_{0}, \boldsymbol{h}_{\mathbf{1}}$ such that $\boldsymbol{h}_{0} \cap \boldsymbol{h}_{1}=\mathbf{0}$ and $\boldsymbol{h}_{0} \cup \boldsymbol{h}_{1}=\mathbf{0}^{\prime}\left({ }^{2}\right)$. Although each of the sets $A_{i}$ of Theorem 5 is generic for $\mathscr{L}_{1}^{*}$, there is no reason to believe that the pair of them is generic for the language with two pairs of adjoined constants.

2.7. Conclusion. We believe that the forcing method will continue to be a useful tool for constructing sets with specified properties of recursion-theoretic interest, and thus it would seem worthwhile to understand the method, in its full generality, in recursion-theoretic terms. It appears to us that one of the most significant ways of classifying the forms of the method is according to the strength of the language $(\mathscr{L})$ utilized together with the complexity of the intended interpretations of the adjoined symbols $\left(\boldsymbol{A}, \boldsymbol{A}^{\prime}\right)$. Thus the form used in $[\mathrm{F}, \S 2]$ is quite weak;

$\left({ }^{2}\right)$ Mr. Joseph Harrison has recently shown that such hyperdegrees exist. His proofs do not use any forcing method. 
it is strengthened in [F, §3] by strengthening the underlying language, and in the present $\$ \S 1.3-1.6$ by using adjoined symbols having more complex interpretations.

As a first estimate, one would expect the languages appropriate to the problems of $\S \S 2.2-2.6$ to be, with the adjoined symbols, just strong enough to express such statements as $A \leq_{h} B$. The languages actually used were plainly stronger. This explains the fact that the solutions obtained were better than required (cf. \$2.1). But if the methods were too coarse, then one would expect to be able to solve the problem posed in $\$ 2.2$ only under an assumption stronger than " $0<\operatorname{hyp}(B)$ " (perhaps " $B$ is not arithmetically definable from $N^{\prime}$ ”). This is a matter which should be cleared up by a general theory of forcing.

\section{REFERENCES}

A $_{1}$. J. W. Addison, Some consequences of the axiom of constructibility, Fund. Math. 46 (1959), 337-357.

$\mathrm{A}_{2}$. - The undefinability of the definable, Abstract 622-71, Notices Amer. Math. Soc. 12 (1965), 347.

C. Paul J. Cohen, The independence of the continuum hypothesis, Proc. Nat. Acad. Sci. U.S.A. 50 (1963), 1143-1148.

F. Solomon Feferman, Some applications of the notions of forcing and generic sets, Fund. Math. 56 (1965), 325-345.

F-S. S. Feferman and C. Spector, Incompleteness along paths in progressions of theories, J. Symbolic Logic 27 (1962), 383-390.

H. P. G. Hinman, Generalizations of some standard theorems on recursive functions, Abstract 65T-238, Notices Amer. Math. Soc. 12 (1965), 466.

$\mathbf{K}_{1}$. S. C. Kleene, Arithmetical predicates and function quantifiers, Trans. Amer. Math. Soc. 79 (1955), 312-340.

$\mathbf{K}_{2}$. - On the forms of predicates in the theory of constructive ordinals. II, Amer. J. Math. 77 (1955), 405-428.

K-R. D. L. Kreider and H. Rogers, Constructive versions of ordinal number classes, Trans. Amer. Math. Soc. 100 (1961), 325-369.

Kr. G. Kreisel, The axiom of choice and the class of hyperarithmetic functions, Indag. Math. 24 (1962), 307-319.

S. C. Spector, Recursive well-orderings, J. Symbolic Logic 20 (1955), 151-163.

$\mathbf{T}_{1}$. S. K. Thomason, Hyperarithmetic incomparability, Abstract 624-3, Notices Amer. Math. Soc. 12 (1965), 449.

$\mathbf{T}_{2 .}-$, The iterated hyperjump, Abstract 625-130, Notices Amer. Math. Soc. 12 (1965), 583.

$\mathbf{T}_{3}$. - The forcing method and the upper semilattice of hyperdegrees, Ph.D. Thesis, Cornell Univ., Ithaca, New York, 1966.

$\mathbf{T}_{4} .-$, Lattice-embeddings in the degrees and hyperdegrees, Abstract 65T-35, Notices Amer. Math. Soc. 13 (1966), 136.

SiMON FrASER UNIVERSITY, Burnaby, B. C., CANADa 